\title{
Primer reporte de forrajeo sobre el chanchito de mar (Emerita analoga) por perros (Canis lupus familiaris) en la costa de Valdivia, Chile
}

\author{
First report of foraging behaviour on Pacific molecrabs (Emerita analoga) by dogs (Canis lupus \\ familiaris) on the coast of Valdivia, Chile
}

\section{Sara M. Rodríguez ${ }^{1, *}$, Katherine Burgos ${ }^{1}$, Bárbara Gutiérrez ${ }^{1}$, Valentina Escares ${ }^{1}$ \& James E. Byers $^{2}$}

${ }^{1}$ Instituto de Ciencias Marinas y Limnológicas, Facultad de Ciencias, Universidad Austral de Chile, Valdivia, Chile.

${ }^{2}$ Odum School of Ecology, University of Georgia, Athens, GA 30602, USA.

*E-mail: saramrodriz@gmail.com

\section{RESUMEN}

Perros domésticos son reportados forrajeando sobre el "chanchito de mar" Emerita analoga en la playa Curiñanco, Valdivia, Chile. Consumir estos crustáceos representa un potencial riesgo para la salud de ellos, ya que E. analoga alberga larvas del acantocéfalo Profilicollis altmani, el cual usa a las aves como hospedador definitivo, pero que también parasita a mamíferos marinos, terrestres e incluso a humanos. Finalmente, los perros no sólo interrumpen el forrajeo de las aves, sino que ahora compiten por el mismo recurso.

Palabras clave: Acanthocephala, playa Curiñanco, Profilicollis altmani.

\section{ABSTRACT}

Domestic dogs are reported foraging on molecrabs Emerita analoga on Curiñanco beach, Valdivia, Chile. Preying on these crabs represents a potential health risk for the dogs, because E. analoga is the intermediate host of larvae of the acanthocephalan Profilicollis altmani, which uses shorebirds as definitive hosts, but also have been documented to parasitize marine and terrestrial mammals including humans. Finally, the dogs not only disrupt birds' foraging, but now also compete for the same resource.

Keywords: Acanthocephala, Curiñanco beach, Profilicollis altmani.

Las playas de arena son uno de los ecosistemas con mayor extensión en el planeta. Estos hábitats se caracterizan por albergar una amplia diversidad de especies de invertebrados, los cuales son una fuente de alimento de múltiples vertebrados, especialmente de aves (McLeod et al. 2013; Schlacher et al. 2015). En el sur de América del Sur, una de las especies clave y más abundante de este ecosistema es el comúnmente conocido como chanchito de mar, el decápodo Emerita analoga (Stimpson, 1857), el cual se distribuye a lo largo de la costa del Océano Pacífico, desde Alaska $\left(58^{\circ} \mathrm{N}\right)$ hasta las costas del Sur de Chile $\left(55^{\circ} \mathrm{S}\right.$; Contreras et al. 1999;
Smith 2007). E. analoga ha sido descrita como alimento tanto de peces como de distintas aves de la familia Laridae, siendo un eslabón esencial en las redes tróficas de estos ecosistemas (Blokpoel et al. 1992; Licandeo et al. 2006; Rodríguez et al. 2017; Rodríguez \& Valdivia 2017). Dentro de sus depredadores está la gaviota Garuma Leucophaeus modestus (Tschudi, 1843), la cual ha sido indicada como especialista en el consumo de este decápodo, abarcando más del $90 \%$ de su dieta (Blokpoel et al. 1992; Rodríguez et al. 2017). Esta gaviota suele escarbar la arena y enterrar su pico en la zona de la rompiente de la ola (swash zone) para capturar los chanchitos de mar (Obs. Pers 
SMR). L. modestus migra en época estival a las costas de la Región de Los Ríos y se alimenta activamente de E. analoga, para luego regresar a su hábitat reproductivo en el Norte de Chile (Guerra et al. 1988; Aguilar et al. 2016). Sin embargo, poblaciones de individuos juveniles han sido observado como residentes en distintos puntos de la costa de la Región de los Ríos desde el año 2014 (Rodríguez \& Valdivia 2017). Sumado a L. modestus, otras especies de aves, incluyendo la gaviota Cáhuil Chroicocephalus maculipennis Lichtenstein, 1823, la gaviota Dominicana Larus dominicanus Lichtenstein, 1823, la gaviota Franklin Leucophaeus pipixcan (Wagler, 1831), el Zarapito común Numenius phaeopus (Linnaeus, 1758), y el playero Calidris alba Palla, 1764, son frecuentes en esta costa y también depredan sobre de $E$. analoga. Pero al contrario de L. modestus, estas especies depredan principalmente sobre individuos presentes en la zona del espejo (effluent line) de la playa (Obs. Pers. SMR). Por lo tanto, disturbios que puedan generarse en estos hábitats, afectarían directamente en la presencia y actividades de forrajeo de estas aves marinas.

La actividad antrópica en las áreas costeras ha sido catalogada como un "disturbio" que altera las actividades normales o fisiológicas de la vida salvaje (McLeod et al. 2013; Altman \& Byers 2014). La presencia de caminantes, recolectores de algas varadas y pescadores genera múltiples efectos adversos sobre la fauna que habita en estos lugares (Cornelius et al. 2001; Meager et al. 2012; Navedo et al. 2019). Sumado a esto, la presencia humana suele estar acompañada de perros (Canis lupus familiaris Linnaeus, 1758), los cuales provocan efectos aún más pronunciados (Randler 2006). En este sentido, las aves marinas son las especies que reciben la mayor cantidad de impactos por la presencia de los perros (Lafferty 2001; Randler 2006; Navedo et al. 2019). Efectos en la abundancia de aves, frecuencia y calidad del forrajeo y descanso han sido algunas de las actividades que son afectadas negativamente (Keller 1989; McLeod et al. 2013; Navedo et al. 2019). Además, los perros son catalogados como depredadores tope, los cuales consumen fauna silvestre, en especial huevos y crías de distintas especies de aves (Keller 1989; Sime 1999). Sin embargo, son escasos los reportes y/o estudios en que muestran a animales domésticos depredando activamente sobre recursos de origen marino, y son casi inexistentes registros del uso de técnicas como escarbar para atraparlos (Schlacher et al. 2015). Por lo tanto, es relevante el analizar el efecto que pueda ejercer la presencia y la actividad que realizan los perros en estas zonas naturales. En este trabajo reportamos a la playa de arena como nuevo potencial hábitat de forrajeo para perros domésticos y al crustáceo E. analoga como parte de la extensión de su dieta. Además, discutimos sobre los efectos que conlleva está actividad en estos hábitats; y los costos y beneficios de esta nueva conducta adquirida por los perros.

Durante trabajos en terreno en marea baja en la playa Curiñanco, Valdivia $\left(-39,81^{\circ} \mathrm{S}-73,24^{\circ} \mathrm{O}\right.$; Fig. $1 \mathrm{~A}$ y $\left.1 \mathrm{~B}\right)$ el 28 de Agosto de 2019, se encontraron zonas con hoyos y arena removida (Fig. 2). Dicho disturbio era causado por tres perros domésticos (C. lupus familiaris) que escarbaban la arena enérgicamente, principalmente en la zona del efluente (effluent line). Observamos que luego de escarbar, olfateaban la arena, la seguían removiendo con el hocico y capturaban individuos de E. analoga, los que luego eran comidos. Esta actividad fue observada en reiteradas ocasiones en distintos lugares alrededor de la zona del efluente de la playa por un período de 45 minutos aproximadamente (ver video, Mat. Sup. https://gayana.cl/public/MatSup/v84n2/173_MatSupl-S1.mp4). Desde ese día, hemos observado esa conducta en el mismo lugar y en otros sectores de la misma playa de Curiñanco. Por ejemplo, esta conducta fue observada el 12 de Septiembre de 2019 y el 6 de Noviembre de 2019 por los mismos tres perros, y el 4 de Diciembre de 2019 por parte de dos de dichos perros.

En una visita realizada a la playa durante marea alta el 12 de Diciembre de 2019, preguntamos a cinco pescadores que se encontraban a orilla de la playa, si es que habían observado la conducta de forrajeo aquí descrita. Dos de ellos afirmaron que esa conducta la han observado en reiteradas ocasiones por lo menos desde hace un año. También aseveraron haber visto a otros perros realizando la misma actividad. Sin embargo, ninguno de estos pescadores se adjudicaron ser los dueños de estos animales. Dado los registros y observaciones realizadas en terreno, podemos concluir que esta sería una conducta que probablemente ya está adoptada por los perros y que sería usada por ellos para mejorar su alimentación.

En las playas de Chile es común la presencia de pescadores en compañía de perros (Navedo et al. 2019). En la playa Curiñanco, los pescadores utilizan frecuentemente a $E$. analoga como cebo para la pesca, en especial del róbalo Eleginops maclovinus (Cuvier \& Valenciennes 1830), dado que es una presa recurrente en la dieta de estos peces (Licandeo et al. 2006). Probablemente, los perros han aprendido e imitado la forma de captura de E. analoga, cuando los pescadores escarban el sustrato para extraerlas de la arena. La abundancia y facilidad con la que se captura $E$. analoga, y el alto contenido de lípidos y proteínas que poseen los decápodos en su musculatura (Oliveira et al. 2007), los transforman en un buen recurso para la dieta de los perros (Sime 1999). Junto con esto, las perturbaciones que realizan los perros en estos hábitats son aún más intensas si es que estos animales no sólo están de paso, sino que además utilizan los recursos que son clave en la dieta de individuos costeros, como son las aves. Diversas especies de aves migratorias y residentes se alimentan de 
E. analoga y de otras especies de macroinvertebrados que habitan en esta zona (Blokpoel et al. 1992; Aguilar et al. 2016; Rodríguez \& Valdivia 2017). De la misma forma, Lafferty et al. (2014) registró que el Pato Azulón Anas platyrhinchos, ave típica de humedales y lagos, frecuentaba la costa de California para forrajear sobre E. analoga. Junto con ese reporte, observó a otras especies de la familia Anatidae alimentándose frecuentemente de este crustáceo, por lo cual pareciera ser una fuente de alimento con un gran valor energético e importante para los animales que la descubren. Por lo tanto, la presencia frecuente y activa de los perros generaría alteraciones en las actividades típicas de las aves, en especial las de alimentación (Navedo et al. 2019).
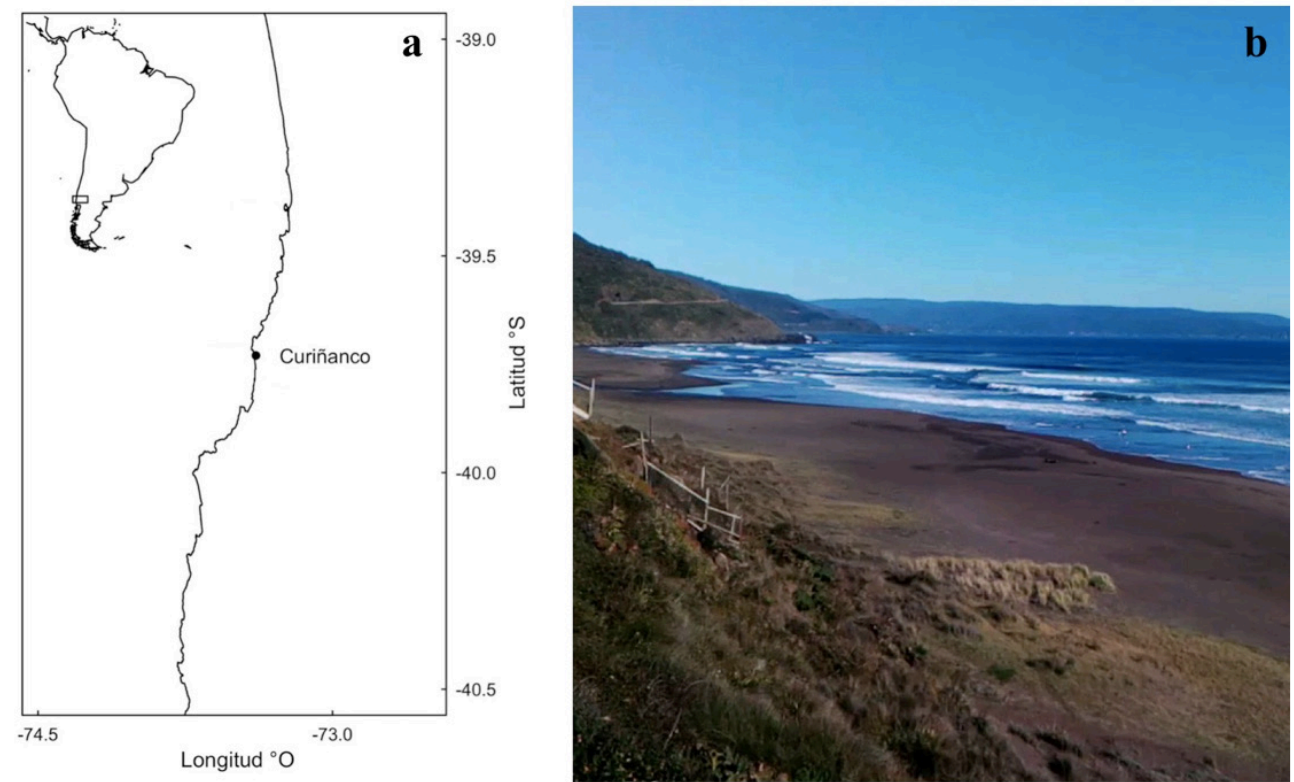

Figura 1. Sitio de estudio: (a) Mapa de la playa Curiñanco, Valdivia, Chile. (b) vista de la playa de Curiñanco mostrando la amplitud de la marea baja. Ancho = 65 m aproximadamente. / Study site: (a) Map of Curiñanco beach, Valdivia, Chile. (b) view of Curiñanco beach showing the low tidal range. Width = approximately $65 \mathrm{~m}$.



Figura 2. Actividades realizadas por los perros en Playa Curiñanco: (a) perros excavando en la arena en busca de Emerita analoga (b) Hoyos en la arena dejados por los perros en la playa. / Activities performed by dogs in Curiñanco beach: (a) dogs digging in the sand in search of Emerita analoga (b) holes in the sand made by dogs on the beach. 
La expansión en la dieta de los perros aquí reportada traería consigo riesgo potencial de infección parasitaria. E. analoga es hospedador intermediario de parásitos helmintos en estado larval como el nemátodo Proleptus sp., el digeneo Maritrema sp. y el acantocéfalo Profilicollis altmani (Smith 2007; Oliva et al. 2008; Rodríguez et al. 2017). Estudios sobre la abundancia de E. analoga en las playas de arena del sur de Chile, registraron que varía estacionalmente, alcanzando el peak promedio de más de 1.600 individuos $\mathrm{x} \mathrm{m2}$ durante época estival (Contreras et al. 1999). En relación al parasitismo en $E$. analoga, se ha reportado que la prevalencia de P. altmani incrementa con el tamaño corporal del hospedador y que varía entre el $7 \%$ (<15 mm de longitud cefalotoráxica; LCT) y el $80 \%$ (>20 mm LCT) (Balboa et al. 2009; Rodríguez \& Valdivia 2017). Además, los parásitos Maritrema sp. y $P$. altmani alcanzan su madurez sexual en distintas especies de gaviotas. Sumado a esto, P. altmani tiene la característica de provocar perforaciones y daño en el intestino de las aves y nutrias (Enhydra lutris nereis) (Mayer et al. 2003; Rodríguez et al. 2017). Infecciones experimentales realizadas con este acantocéfalo indican que parasita exitosamente a otros vertebrados como ratones, gallinas y perros (Tantaleán et al. 2002, 2005). Además, ha sido detectado en humanos que consumen este crustáceo sin cocer, como parte de "ceviche", generando problemas de salud gastrointestinal (Ibáñez \& Machado 1991; Tantaleán et al. 2002, 2005). Por lo tanto, esta frecuente conducta de forrajeo por parte de los perros podría traer consecuencias negativas en su salud, probablemente irreversibles y a la larga tal vez mortales. Adicionalmente, dado que el comportamiento es, aparentemente, transmitido socialmente, esta conducta podría extenderse a otros perros que son recurrentes en las playas de arena de la costa valdiviana. Si bien pareciera ser una conducta local, dado que no se ha reportado en otras playas, corresponde a un fenómeno que ha sido observado en reiteradas ocasiones a lo largo de un semestre y probablemente ya esté instalado como conducta cotidiana de forrajeo. Como conclusión podemos decir que no es una conducta casual y que dado el alto contenido proteico de $E$. analoga, ésta estaría siendo elegida como un nuevo ítem presa. Si este es un comportamiento que en perros se ha vuelto frecuente, se recomienda comenzar a regular la presencia de estos animales en las playas. De esta forma, disminuirá la perturbación hacia las aves y un foco de riesgo sanitario.

\section{AGRADECIMIENTOS}

Se agradece el apoyo financiero de Fondecyt 3190348; al Odum School of Ecology, Universidad de Georgia, por facilitar dependencias y a la Dra. Claudia Aracena por la ayuda del material audiovisual.

\section{REFERENCIAS}

Aguilar, R., Simeone, A., Rottmann, J., Perucci, M., Luna-Jorquera, G. 2016. Unusual coastal breeding in the desert-nesting Gray gull (Leucophaeus modestus) in Northern Chile. Waterbirds 39(1): 69-73.

Altman, I., Byers, J.E. 2014. Large-scale spatial variation in parasite communities influenced by anthropogenic factors. Ecology 95(7): 1876-1887.

Balboa, L., Hinojosa, A., Riquelme, C., Rodríguez, S., Bustos, J., George-Nascimento, M. 2009. Alloxenic distribution of cystacanths of two Profilicollis species in sympatric crustacean hosts in Chile. Journal of Parasitology 95: 1205-1208.

Blokpoel, H., Boersma, D.C., Hughes, R.A., Tessier, G.D. 1992. Foraging by larids on sand crabs Emerita analoga along the coast of Southern Peru. Ardea 80: 99-104.

Contreras, H., Defeo, O., Jaramillo, E. 1999. Life history of Emerita analoga (Stimpson) (Anomura, Hippidae) in a sandy beach of South central Chile. Estuarine, Coastal and Shelf Science 48: 101-112.

Cornelius, C., Navarrete, S.A., Marquet, P.A. 2001. Effects of human activity on the structure of coastal marine birds assemblages in central Chile. Conservation Biology 15: 1396-1404.

Guerra, C.G., Fitzpatrick, L.C., Aguilar, R.E. 1988. Influence of desert nesting and foraging distance on growth rates in Gray gulls (Larus modestus). Auk 105: 779-783.

Ibáñez, H.N., Machado, F.D.A. 1991. Infección humana con Falsificollis sphaerocephalus (Acanthocephala) en Trujillo, Perú. Revista Peruana Médica Tropical UNMSM 5: 75-78.

Keller, V. 1989. Variation in the response of great crested grebes Podiceps cristatus to human disturbance-a sign adaptation? Biological Conservation 49: 31-45.

Lafferty, K.D. 2001. Birds at a Southern California beach: seasonality, hábitat use and disturbance by human activity. Biodiversity and Conservation 10: 1949-1962.

Lafferty, K.D., Mclaughlin, J.P., Dugan, J.E. 2014. Novel foraging in the swash zone on pacific sand crabs (Emerita analoga, Hippidae) by Mallards. The Wilson Journal of Ornithology 125(2): 423-426.

Licandeo, R.R., Barrientos, C.A., González, M.T. 2006. Age, growth rates, sex change and feeding habits of notothenids fish Eleginops maclovinus from the central-southern Chilean coast. Environmental Biology of Fishes 77: 51-61.

Mayer, K.A., Dailey, M.D., Miller, M.A. 2003. Helminths parasites 
of the Southern sea otter Enhydra lutris nereis in central California: abundance, distribution and pathology. Diseases of Aquatic Organisms 53: 77-88.

Mcleod, E.M., Guay, P.J., Taysom, A.J., Robinson, R.W., Weston, M.A. 2013. Buses, cars, bicycles and walkers: the influence of the type of human transportation on the flight responses of waterbirds. PLoS ONE 8(12): e82008.

Meager, J.J., Schlacher, T.A., Nielsen, T. 2012. Humans alter habitat selection of birds on ocean-exposed sandy beaches. Diversity and Distributions 18: 294-306.

Navedo, J.G., Verdugo, C., Rodríguez-Jorquera, I.A., AbadGómez, J.M., Suazo, C.G., Castañeda, L.E., Araya, V., Ruiz, J., Gutiérrez, J.S. 2019. Assesing the effects of human activities on the foraging opportunities of migratory shorebirds in Austral high-latitude bays. PLoS ONE 15: e0212441.

Randler, C. 2006. Disturbances by dogs barking increase vigilance in coots Fulica atra. European Journal of Wildlife Research 52: 265-270.

Oliva, M.E., Barrios, I., Thatje, S., Laudien, J. 2008. Changes in prevalence and intensity of infection of Profilicollis altman (Perry, 1942) cystacanth (Acanthocephala) parasitizing the mole crab Emerita analoga (Stimpson, 1857): and El Niño cascade effect? Helgoland Marine Research 62 (Suppl 1): S57-S62.

Oliveira, G.T., Fernandes, F.A., Bueno, A.A.P., Bond-Buckup, G. 2007. Seasonal variations in the intermediate metabolism of Aegla platensis (Crustacea, Aeglidae). Comparative Biochemistry and Physiology, Part A 147: 600-606.
Rodríguez, S.M., D’elía, G., Valdivia, N. 2017. The phylogeny and life cycle of two species of Profilicollis (Acanthocephala:Polymorphidae) in marine hosts off the Pacific coast of Chile. Journal of Helminthology 91(5): 589-596.

Rodríguez, S.M., Valdivia, N. 2017. Mesoscale spatiotemporal variability in a complex host-parasite system influenced by intermediate host body size. PeerJ e3675. DOI: 10.7717/peerj.3675.

Schlacher, T.A., Weston, M.A., Lynn, D., Schoeman, D.S., Huijbers, C.M., Olds, A.D., Masters, S., Connolly, R.M. 2015. Conservation gone to the dogs: when canids rule the beach in small coastal reserves. Biodiversity and Conservation 24: 493-509.

Sime, C.A. 1999. Domestic dogs in wildlife hábitats. In: Joslin G, Youmans $\mathrm{H}$ (Eds.) Effects of recreation on Rocky Mountain wildlife: a review for Montana. Montana Chapter of the Wildlife Society. 307 pp.

Smith, N.F. 2007. Associations between shorebird abundance and parasites in the sand crab, Emerita analoga, along the California coast. Journal of Parasitology 93(2): 265-273.

Tantaleán, M., Cárdenas, J., Güere, R. 2002. Profilicollis altmani (Perry, 1942) Van Cleave, 1947 (Acanthocephala) en el Perú. Con notas sobre la infección experimental de mamíferos marinos. Revista Peruana de Biología 9(1): 49-51.

Tantaleán, M., Sánchez, L., Gómez, L., Huiza, A. 2005. Acantocéfalos del Perú. Revista Peruana de Biología 12(1): 83-92

Received: 02.03.2020

Accepted: 14.07.2020 